SESSION 7: Lessons from local galaxies and high-z analogues 


\title{
Analogs of high redshift galaxies: Disentangling the complexity of the green peas
}

\section{Ricardo Amorin}

University of La Serena, Chile

\begin{abstract}
Young low-mass galaxies with extreme emission-line properties are ubiquitous at high redshift. However, a detailed characterisation of their physical properties, key for understanding cosmic reionisation and the early growth of galaxies, will be only possible with JWST and ELT observations. Rare lower-z analogues of these primeval galaxies provide us ideal laboratories to study in larger detail the complex physical mechanisms taking place in these extreme systems. In this talk, I will review key results from these high-z analogues, with an emphasis on lessons learned from deep spectroscopic observations of green pea galaxies at $z \lesssim 0.3$. New recent results based on high-dispersion Echelle and IFU spectroscopy of green peas will be presented. They illustrate current advantages and limitations of the chemodynamical analysis for a simultaneous study of the ionised gas kinematics, chemical enrichment and the escape of ionising photons in compact low-mass starbursts.
\end{abstract}

